ARTIGO ORIGINAL

\title{
Síndrome de Burnout em médicos residentes
}

\section{Burnout syndrome in resident physicians}

Natália Martins Falcão ${ }^{1}$. Eugênio de Moura Campos² ${ }^{2}$ Dafne de Albuquerque Simão ${ }^{3}$. Nathália Silva Sena². Mônica Taynara Muniz Ferreira².

1 Hospital de Saúde Mental Frota Pinto, Fortaleza, Ceará, Brasil. 2 Universidade Federal do Ceará (UFC), Fortaleza, Ceará, Brasil. 3 Hospital Universitário Walter Cantídio (HUWC), Fortaleza, Ceará, Brasil.

\section{RESUMO}

A Síndrome de Burnout caracteriza-se pela tríade de exaustão emocional, baixa realização profissional e sentimentos de desrealização. Objetivo: analisar a prevalência da Síndrome de Burnout e aspectos relacionados em médicos residentes do Hospital Universitário Walter Cantídio no período de novembro a dezembro de 2017. Metodologia: trata-se de estudo quantitativo e descritivo, com coleta de dados a partir de questionários estruturados aplicados com 158 médicos residentes. Os resultados foram analisados estatisticamente utilizando o dispositivo SPSS 22.0, sendo realizados os testes do Qui-Quadrado de Pearson e o Teste Exato de Fisher. Resultados: os resultados apontam que 68,7\% (101) dos residentes apresentam níveis elevados de Síndrome de Burnout. Através da Escala de Sonolência Diurna de Epworth, identificamos que 19,7\% trazem como resultado índice muito patológico. Observamos que não há influência estatística significativa do sexo $(\mathrm{p}=0,637)$ e da especialidade ( $\mathrm{p}=0,127)$ na incidência da Síndrome de Burnout e que não há associação entre portadores de Síndrome de Burnout Grave e índices elevados de sonolência diurna $(\mathrm{p}=0,340)$. Conclusão: conclui-se que os médicos residentes apresentam grau elevado de Síndrome de Burnout e altos índices patológicos de sonolência diurna. Assim, faz-se necessário mudanças nessa modalidade de formação.

Palavras-chave: Esgotamento profissional. Educação continuada. Internato e residência. Recursos humanos.

\section{ABSTRACT}

Burnout Syndrome is characterized by the triad of emotional exhaustion, low professional achievement and feelings of derealization. Objective: analyze the prevalence of Burnout Syndrome and related aspects in resident physicians of Hospital Universitário Walter Cantídio from November to December 2017. Methodology: it is a quantitative and descriptive study, with data collection from structured questionnaires applied with 158 resident physicians. The results were analyzed statistically using the SPSS 22.0 device, with the Pearson Chi-Square tests and the Fisher's Exact Test. Results: the results indicate that 68.7\% (101) of the residents have high levels of Burnout Syndrome. Through the Epworth diurnal sleepiness scale, we found that in 19.7\% they bring as a result very pathological index. We observed that there was no significant statistical influence of the $\operatorname{sex}(p=0.637)$ and the specialty $(p=$ 0.127) on the incidence of Burnout Syndrome and that there was no association between Severe Burnout Syndrome and elevated daytime sleepiness indexes $(\mathrm{p}=0,340)$. Conclusion: it is concluded that resident physicians have a high degree of Burnout Syndrome and high pathological indices of daytime sleepiness. Thus, changes in this type of training are necessary.

Keywords: Burnout, professional. Education, continuing. Internship and residency. Human resources.

Autor correspondente: Natália Martins Falcão, Avenida Oliveira Paiva,1090, Cidade dos Funcionários, Fortaleza, Ceará. CEP: 60822-131. Telefone: +5585 99505-5650. E-mail: nataliamfalcao@gmail.com

Conflito de interesses: Não há qualquer conflito de interesses por parte de qualquer um dos autores.

Recebido em: 27 Ago 2018; Revisado em: 16 Out 2018; Aceito em: 07 Nov 2018. 


\section{INTRODUÇÃO}

A Síndrome de Burnout, conforme o próprio nome sugere, consiste em um estado de exaustão física e mental, decorrente da vida profissional. A rigor, constitui-se a partir de três dimensões predominantemente afetadas no indivíduo: exaustão emocional, despersonalização e baixos níveis de realização pessoal no trabalho. ${ }^{1}$

Não existe padrão único, rígido e fixo acerca do diagnóstico e tratamento da doença. Não temos, por exemplo, sua presença codificada nas versões mais atuais do Código Internacional de Doenças (CID-10), bem como do Manual de Diagnóstico e Estatística dos Transtornos Mentais (DSM-V) $)^{2,3}$ como uma entidade específica. A síndrome vem sendo considerada na CID-10 como pertencente ao diagnóstico de Transtorno de Adaptação (F43.2) e/ou problemas relacionados com o emprego e desemprego (Z56).

O diagnóstico da Síndrome de Burnout é eminentemente clínico, entretanto, os estudos realizados sobre o tema utilizam como suporte ao diagnóstico o emprego de instrumentos que auxiliam também na tomada de decisão quanto ao projeto terapêutico.

Um agravante da Síndrome de Burnout, principalmente no que diz respeito ao aumento nos níveis de exaustão, é a qualidade do sono. Esta síndrome associada aos distúrbios do sono pode provocar diversas consequências sérias ao indivíduo, como transtorno de ansiedade, depressão, abuso de drogas, pensamentos suicidas, abandono, baixa motivação para o aprendizado e baixo desempenho acadêmico. ${ }^{4}$

Dentre os profissionais, sabe-se que os médicos estão diariamente expostos a uma grande carga de estresse, especialmente no período da Residência Médica. Existem poucos estudos no Brasil direcionados aos profissionais de saúde. O conhecimento minucioso deste tema é relevante visto que podem ocorrer graves injúrias aos profissionais portadores deste transtorno que não são diagnosticados e tratados precocemente. Nesse sentido, o presente estudo tem por objetivo analisar a prevalência da Síndrome de Burnout e aspectos relacionados em médicos residentes do Hospital Universitário Walter Cantídio no período de novembro a dezembro de 2017.

\section{METODOLOGIA}

Trata-se de estudo quantitativo e descritivo, com coleta de dados a partir de questionários estruturados. A pesquisa foi realizada entre os médicos residentes do Hospital Universitário Walter Cantídio no período de novembro a dezembro de 2017. Os sujeitos da pesquisa responderam a um caderno de resposta auto informativo, não identificado, contendo um questionário sociodemográfico, além do Inventário de Burnout de Maslach e da Escala de Sonolência Diurna de Epworth. O projeto foi aprovado pelo Comitê de Ética em Pesquisa do Hospital Universitário Walter Cantídio e esteve de acordo com os critérios estabelecidos pela Declaração de Helsinki. Os resultados foram codificados e tabelados e posteriormente analisados estatisticamente utilizando o dispositivo SPSS 22.0, sendo realizados os Testes do Qui-Quadrado de Pearson e o Teste Exato de Fisher.

\section{RESULTADOS}

Do total de 206 residentes, 76,6\% (158) responderam os questionários. Destes, temos que 97 são do sexo feminino e 61 do masculino. Com relação às grandes áreas de especialização, obtivemos a seguinte contagem: 17 residentes de Pediatria, 82 de Clínica Médica e 39 de Clínica Cirúrgica. Residentes de ginecologia e obstetrícia não foram incluídos neste trabalho.

Os resultados apontam que 68,7\% (101) dos residentes apresentaram níveis elevados de Síndrome de Burnout. Destes $69,5 \%$ (41) são homens, e 68,2\% (60) mulheres. Ao avaliarmos os dados do nosso estudo através do teste do Qui-quadrado de Pearson e do Teste Exato de Fisher, observamos que não há influência estatística significativa do sexo $(p=0,637)$ e da especialidade $(\mathrm{p}=0,127)$ na incidência da Síndrome de Burnout. Encontramos também, que não há associação entre portadores de Síndrome de Burnout Grave e índices elevados de sonolência diurna $(\mathrm{p}=0,340)$, ou seja, residentes portadores de sonolência diurna elevada não apresentam maior incidência de Síndrome de Burnout Grave do que a população de residentes geral.

Analisando os dados obtidos através da Escala de Sonolência Diurna de Epworth, identificamos que 43,5\% dos residentes apresentam índices de sonolência diurna considerados normais, $36,7 \%$ apresentam níveis patológicos e 19,7\% trazem como resultado índice muito patológico. Ao avaliarmos a Síndrome de Burnout e a sonolência diurna conjuntamente, constatamos que não há associação entre estas variáveis $(\mathrm{p}=0,34)$. Nesse sentido, são apresentadas a seguir as Tabelas 1 e 2. Quando aferimos conjuntamente os índices de sonolência diurna elevada e o sexo, encontramos uma associação positiva $(\mathrm{p}=$ 0,004), ou seja, estatisticamente, homens apresentam índices maiores de sonolência diurna elevada do que as mulheres. Aferimos também que não há associação entre a especialidade e o índice elevado de sonolência diurna $(\mathrm{p}=0,655)$.

Tabela 1. Síndrome de Burnout e sonolência diurna correlacionadas com o sexo.

\begin{tabular}{cccc}
\hline Condição de saúde & Masculino & Feminino & P \\
\hline Síndrome de Burnout Grave & $60(68,2 \%)$ & $41(69,5 \%)$ & 0,637 \\
$\begin{array}{c}\text { Sonolência Diurna Muito } \\
\text { Patológica }\end{array}$ & $27(27,3 \%)$ & $6(8,5 \%)$ & 0,004 \\
\hline
\end{tabular}

Tabela 2. Síndrome de Burnout e sonolência diurna correlacionadas com a especialidade.

\begin{tabular}{ccccc}
\hline Condição de saúde & Clínica & Cirúrgica & Pediátrica & P \\
\hline $\begin{array}{c}\text { Síndrome de } \\
\text { Burnout Grave }\end{array}$ & $50(61 \%)$ & $29(74,4 \%)$ & $14(82,4 \%)$ & 0,127 \\
$\begin{array}{c}\text { Sonolência Diurna } \\
\text { Muito Patológica }\end{array}$ & $14(17,1 \%)$ & $7(17,9 \%)$ & $6(35,3 \%)$ & 0,655 \\
\hline
\end{tabular}




\section{DISCUSSÃO}

A residência médica trata-se de um período de extrema sobrecarga física e emocional para o médico residente. Além de ser um período em que é necessário um grande número de horas de estudo, o médico é forçado a desenvolver rapidamente as novas habilidades da especialidade e passa a lidar com longas jornadas de trabalho, estando responsável pela vida de paciente potencialmente graves, sendo submetido a pressões internas e sociais que levam ao médico a exigência de atingir um ideal irreal de perfeição. Somado a isso, tem-se a baixa remuneração financeira que por vezes leva o residente a utilizar o seu possível tempo livre em outras atividades de trabalho, visando uma melhor remuneração financeira e culminando numa carga de trabalho e estresse ainda maiores.

As longas jornadas de trabalho, o pouco tempo para descanso, a cobrança de preceptores e pacientes levam ao aumento da responsabilidade profissional e a diminuição do tempo para vida social. $^{5}$

A avaliação da prevalência da síndrome de Burnout não demonstrou diferença significativa entre homens e mulheres, o que contradiz a literatura. Normalmente, mulheres apresentam níveis mais elevados em escalas que avaliam ansiedade e depressão. Um estudo da Escola Paulista de Medicina de 1998 avaliou o potencial de diversas situações ansiogênicas que ocorriam com os médicos residentes, encontrando que as mulheres apresentavam maiores escores em nível de ansiedade antecipatória que os homens. Este mesmo estudo, ao discutir a etiologia deste quadro sindrômico, traz as seguintes situações como principais fatores etiológicos: privação de sono, excessiva carga de trabalho, responsabilidade profissional, mudanças frequentes das condições de trabalho e a competição entre os residentes. ${ }^{6}$

Os valores equiparados da síndrome de Burnout entre homens e mulheres neste estudo e a ausência de associação estatística entre Síndrome de Burnout e o sexo podem ser decorrentes de ambos os sexos estarem submetidos às mesmas obrigações durante a residência médica. Em seu artigo, Assaiag 5 avalia a possibilidade de as mulheres médicas se obrigarem a assumir uma postura semelhante a do sexo masculino durante o trabalho, modificando sua posição com relação à própria saúde e a qualidade de vida, vindo daí a similaridade da prevalência da síndrome de Burnout entre os sexos.

Com relação à sonolência diurna, os homens apresentaram índices patológicos de $27,3 \%$, porcentagem bem maior que as mulheres, que pontuaram somente $8,5 \%$, ocorrendo associação positiva entre sonolência diurna e o sexo $(\mathrm{p}=0,004)$. Estes

\section{REFERÊNCIAS}

1. Freudenberger HJ. Staff burn-out. J Soc Issues. 1974;30(1):15965 .

2. American Psychiatric Association. Diagnostic and statistical manual of mental disorders (DSM-5®). [place unknown]: American valores estão de acordo com a literatura que demonstra que homens apresentam postura de risco com relação à própria saúde, levando mais tempo para procurarem auxílio médico especializado. $^{7}$

Em nosso estudo, a presença de síndrome de Burnout grave foi caracterizada quando da existência de escores elevados em qualquer um dos três domínios (exaustão emocional, despersonalização ou baixa realização pessoal), conforme orientado pelos autores do Inventario de Maslach, e seguindo a Tabela 3, conforme apresentada a seguir.

Tabela 3. Range dos escores indicando o nível de Burnout por subescala.

\begin{tabular}{cccc}
\hline Subescala & Baixo & Moderado & Alto \\
\hline Exaustão emocional & $0-16$ & $17-26$ & $27+$ \\
Despersonalização & $0-6$ & $07-12$ & $13+$ \\
Realização pessoal & $39_{+}$ & $32-38$ & $0-31$ \\
\hline
\end{tabular}

Em relação à prevalência da Síndrome de Burnout, 68,7\% (101) dos residentes apresentaram níveis elevados. Com relação à presença de Síndrome de Burnout e Sonolência Diurna Patológica, os maiores valores foram encontrados na especialidade de Pediatria, entretanto, após análise estatística, não se encontrou associação entre Síndrome de Burnout e especialidade médica $(\mathrm{p}=0,127)$ e nem entre sonolência diurna e especialidade médica ( $p=0,655)$ o que traz um achado diferente dos estudos nacionais. $\mathrm{Na}$ maioria dos estudos nacionais e internacionais, a especialidade com índices mais elevados de Síndrome de Burnout e de sonolência diurna patológica são as especialidades cirúrgicas.

\section{CONCLUSÕES}

A maioria dos médicos residentes percebe o período da Residência Médica como uma etapa de suas vidas associada à baixa qualidade de vida e a altos níveis de estresse físico e emocional. O grande número de residentes apresentando grau elevado de Síndrome de Burnout e índices patológicos de sonolência diurna demonstra que faz-se necessário mudanças no modo de treinamento da residência médica, bem como a disponibilização de uma equipe de suporte psicossocial ao médico residente visto que a presença da Síndrome de Burnout e da sonolência diurna patológica podem acarretar danos não só aos médicos em formação, mas também aos pacientes, cuidados por profissionais adoentados. Mais pesquisas sobre este tema se fazem necessárias.

Psychiatric Pub; 2013 [Acesso em: 20 ago 2017]. Disponível em: https://www.psychiatry.org/psychiatrists/practice/dsm

3. World Health Organization. International statistical classification of diseases and related health problems. [place unknown]: World 
Health Organization; 2004 [Acesso em: 20 ago 2017]. Disponível em: https://www.cid10.com.br/

4. Pagnin D, Queiroz V. Influence of burnout and sleep difficulties on the quality of life among medical students. Springerplus. 2015;4(1):676.

5. Asaiag PE, Perotta B, Martins MD, Tempski P. Avaliação da qualidade de vida, sonolência diurna e burnout em médicos residentes. Rev Bras Educ Med. 2010;34(3):422-9.

6. Martins LA. Natureza e magnitude do estresse na residência médica. Rev Assoc Med Bras. 1998;44(1):28-34.

7. Dickstein LJ, Stephenson JJ, Hinz LD. Psychiatric impairment in medical students. Acad Med. 1990;65(9):588-93.

\section{Como citar:}

Falcão NM, Campos EM, Simão DA, Sena NS, Ferreira MT. Síndrome de Burnout em médicos residentes. Rev Med UFC. 2019 jul-set;59(3):20-23. 